\title{
Fecal Microbiota Transplantation Is Effective for the Treatment of Partially Treated Clostridioides difficile Infection
}

\author{
Young-Seok Cho \\ Department of Internal Medicine, Seoul St. Mary's Hospital, College of Medicine, The Catholic University of Korea, Seoul, Korea
}

\author{
Corresponding Author \\ Young-Seok Cho \\ ORCID https://orcid.org/0000-0003-1537-3427 \\ E-mail yscho@catholic.ac.kr
}

\author{
See "Factors Related to Outcomes of Fecal Microbiota Transplantation in Patients with \\ Clostridioides difficile Infection" by Hyuk Yoon, et al. on page 61, Vol. 15, No. 1, 2021
}

Fecal microbiota transplantation (FMT) is the infusion of feces from healthy donors to restore healthy microbiota. FMT has emerged as highly effective, safe, and costeffective treatment option for recurrent Clostridioides difficile infection (CDI) with a success rate around of $90 \% .{ }^{1}$ In addition, FMT might be an encouraging therapeutic approach for the treatment of refractory, severe or complicated CDI and Clostridioides difficile ribotype 027. ${ }^{2}$ Based on these findings, multiple national and international consensus groups have included FMT as the standard of care for recurrent CDI. ${ }^{3}$ In Korea, there have been a few studies for the usefulness of FMT in this condition.

In the Gut and Liver, Yoon et al. ${ }^{4}$ report clinical outcomes in patients with CDI who underwent FMT. In this study, 20 patients, including $45 \%$ of patients with fulminant CDI, were treated with fresh or frozen FMT. The overall success rate in this cohort was 55\% after 1st FMT and 75\% after 2nd FMT, and showed lower rates compared with those from meta-analyses. These findings may be attributed to inclusion of a number of severe or severe-complicated patients. It is unknown at what time point FMT should be performed. The researchers also performed FMT for the treatment of CDI patients who were not completely resolved after appropriate antibiotics therapy. Surprisingly, FMT had excellent treatment outcomes regardless of other factors in these patients with partially treated CDI, and the success rate was $100 \%$. Studies for the use of FMT for primary CDI are very limited. A retrospective case series including CDI patients with ribotype 027 evaluated the efficacy of early FMT administered via a nasogastric tube within a week of infection in combination with antibiotics in patients with at least three treatment failures or relapses. ${ }^{2}$ The results showed early FMT was associated with significantly reduced mortality compared with using antibiotics only or delayed FMT, and only independent predictive factor of survival. But, 37.5\% of patients undergoing early FMT needed 2nd FMT within a month following 1st FMT due to recurrence. A recent small pilot randomized trial compared the efficacy of FMT administered via enema $(n=9)$ with oral metronidazole $(\mathrm{n}=11)$ as treatment for primary CDI. ${ }^{5}$ Although the study did not meet its primary endpoints, clinical cure observed in $78 \%$ of patients with FMT, compared with $45 \%$ of patients with metronidazole, suggesting that FMT might be an alternative treatment option to antibiotics as treatment of primary CDI. However, we need more studies including a large number of patients for FMT as initial treatment for CDI. Now, a phase 3 trial is underway to evaluate the efficacy of FMT as treatment for primary CDI.

Mechanisms or microbial predictors underlying the efficacy of FMT remain uncertain. The characteristics of microbial profiles in CDI patients successfully treated with FMT include increased microbial diversity after FMT, recovery of community profiles to a level similar to those in the donor stools, decrease in some bacterial taxa including Proteobacteria and Fusobacteria, and increase in Firmicutes and Bacteroidetes. ${ }^{6}$ This study performed microbial analysis using $16 \mathrm{~S}$ ribosomal RNA gene-based amplicon sequencing to determine the gut microbiota of recipients as well as donors. ${ }^{4}$ The results demonstrated changes of the microbial composition of recipient stools after FMT comparable to those from previous studies, 
suggesting that gut community profiles from recipients might predict treatment outcomes of FMT in CDI patients. Although high therapeutic efficacy can make donor selection less important in the treatment of CDI, choosing good donors based on microbial analysis and/or capability of the donor microbial community to recover certain metabolic disturbances related to CDI might be important to improve the efficacy of FMT. Although it is reported to be similar in efficacy or safety between fresh and frozen stools, several studies showed that microbial viability gradually decrease in frozen stool. ${ }^{6}$ International consensus conference on stool banking states that fecal suspensions might safely be stored for up to 2 months at $-20^{\circ} \mathrm{C}$ or for 2 years at $-80^{\circ} \mathrm{C}$ freezer. ${ }^{7}$ This study showed that microbial viability of frozen stool did not decrease for up to 48 weeks in both aerobe and anaerobe cultures, suggesting that frozen stool may be stored for relatively long time. ${ }^{4}$

The active and effective use of FMT may be limited due to insufficient dedicated centers, lack of stool bank, difficulties in recruiting donors, and regulatory issues. Stool banks may offer proper, reliable, timely approach to FMT for CDI, and also facilitate a standardized and economical workflow that guarantees safety and quality of FMT. ${ }^{7.8}$ During the coronavirus disease 2019 (COVID-19) pandemic, we should do our best to avoid the risk of transmitting the SARS-CoV-2 (severe acute respiratory syndrome coronavirus 2) virus. Expert opinions recommend that FMT should be delayed until COVID-19 is better controlled, and can be considered in cases of fulminant CDI without response to maximal combination therapy. The banked stool before December 2019, or stool from coronavirus-negative donor can be used in cases FMT is required. In addition, COVID-19 should be checked in donors and recipients and matched for infection status if pandemic continues. ${ }^{8,9}$ Overall, this study ${ }^{4}$ provides evidence that FMT is highly effective in CDI patients who was not completely resolved after appropriate antibiotics therapy. Further prospective studies including a large number of patients are required.

\section{CONFLICTS OF INTEREST}

No potential conflict of interest relevant to this article was reported.
ORCID

Young-Seok Cho https://orcid.org/0000-0003-1537-3427

\section{REFERENCES}

1. Quraishi MN, Widlak M, Bhala N, et al. Systematic review with meta-analysis: the efficacy of faecal microbiota transplantation for the treatment of recurrent and refractory Clostridium difficile infection. Aliment Pharmacol Ther 2017;46:479-493.

2. Lagier JC, Delord M, Million M, et al. Dramatic reduction in Clostridium difficile ribotype 027 -associated mortality with early fecal transplantation by the nasogastric route: a preliminary report. Eur J Clin Microbiol Infect Dis 2015;34:15971601.

3. Haifer C, Kelly CR, Paramsothy S, et al. Australian consensus statements for the regulation, production and use of faecal microbiota transplantation in clinical practice. Gut 2020;69:801-810.

4. Yoon H, Shim HI, Seol M, et al. Factors related to outcomes of fecal microbiota transplantation in patients with Clostridioides difficile infection. Gut Liver 2021;15:61-69.

5. Juul FE, Garborg K, Bretthauer M, et al. Fecal microbiota transplantation for primary Clostridium difficile infection. N Engl J Med 2018;378:2535-2536.

6. Ng SC, Kamm MA, Yeoh YK, et al. Scientific frontiers in faecal microbiota transplantation: joint document of AsiaPacific Association of Gastroenterology (APAGE) and AsiaPacific Society for Digestive Endoscopy (APSDE). Gut 2020;69:83-91.

7. Cammarota G, Ianiro G, Kelly CR, et al. International consensus conference on stool banking for faecal microbiota transplantation in clinical practice. Gut 2019;68:2111-2121.

8. Ianiro G, Mullish BH, Kelly CR, et al. Reorganisation of faecal microbiota transplant services during the COVID-19 pandemic. Gut 2020;69:1555-1563.

9. Khanna S, Pardi D. Fecal microbiota transplantation for recurrent Clostridioides difficile infection: the COVID-19 era. Am J Gastroenterol 2020;115:971-974. 